\title{
Osteo-Articular Complications in a Pediatric Sickle Cell Disease Population - Imaging Value
}

\author{
Pegado $\mathrm{PF}^{* 1,4}$, da Câmara $\mathrm{RP}^{2}$, Sara $\mathrm{B}^{2}$, Maia $\mathrm{R}^{2}$, Kjollerstrom $\mathrm{P}^{2}$, Petinga $\mathrm{AP}^{3}$, Soares $\mathrm{E}^{3}$ and Alves $\mathrm{P}^{1,4}$ \\ ${ }^{1}$ Radiology Department, Hospital de São José, Centro Hospitalar de Lisboa Central, Lisbon, Portugal \\ ${ }^{2}$ Pediatric Hematology Unit, Pediatrics Department, Hospital D. Estefânia, Centro Hospitalar de Lisboa Central, \\ Lisbon, Portugal \\ ${ }^{3}$ Radiology Department, Hospital D. Estefânia, Centro Hospitalar de Lisboa Central, Lisbon, Portugal \\ ${ }^{4}$ Nova Medical School, Faculdade de Ciências Médicas, Portugal
}

${ }^{*}$ Corresponding author: Pegado PF, Radiology Department, Hospital de São José, Centro Hospitalar de Lisboa Central, Rua. José António Serrano, Lisbon - Portugal 1150-199, Tel: +351 915607834 E-mail: pedropegado@hotmail.com

Citation: Pegado PF, da Câmara RP, Sara B, Maia R, Kjollerstrom P, et al. (2017) Osteo-Articular Complications in a Pediatric Sickle Cell Disease Population - Imaging Value. J Adv Radiol Med Image 2(1): 101. doi: 10.15744/2456-5504.2.101

Received Date: October 26, 2016 Accepted Date: January 06, 2017 Published Date: January 09, 2017

\begin{abstract}
Sickle cell disease (SCD) is the most frequent of a group of conditions known as haemoglobinopathies. The disease is an autosomal recessive genetic disorder, characterized by abnormal haemoglobin (Hemoglobin $\mathrm{S}$ ) that under certain conditions polymerizes resulting in microvascular occlusions.

This pictorial review illustrates the osteo-articular manifestations associated with sickle-cell disease encountered in children in our institution with at least one Haematology appointment in 2013/2014. Osteo-articular manifestations with imaging findings were reported in 28 out of 97 patients. The most frequent complications and those that required hospital care were painful vaso-occlusive crisis and femoral head osteomyelitis.

Simple X-ray, ultrasound (US), computed tomography (CT) and magnetic resonance imaging (MRI) are current modalities that contribute to the diagnosis of osteo-articular disorders in SCD. Marrow hyperplasia, osteomyelitis, medullary and epiphyseal osteonecrosis, growth defects and dactylitis are illustrated.

Knowledge of imaging features of SCD is important for the diagnosis and management of complications in these patients.

Acute osteomyelitis may be particularly difficult to distinguish from bone infarction and MRI could be an important tool in those cases.

Keywords: Sickle cell disease; Osteo-articular complications; Imaging; Osteomyelitis; Bone infarction

List of Abbreviations: SCD: Sickle cell disease; US: Ultrasound; CT: Computed Tomography; MRI: Magnetic Resonance Imaging; Hb: Haemoglobin; VOC: Vaso-occlusive crisis; WI: Weighted Imaging; AVN: Avascular necrosis; RBC: Red blood cell
\end{abstract}

\section{Introduction}

Sickle cell disease is part of a group of genetic disorders known as haemoglobinopathies [1,2]. It results from a mutation in the beta-globin gene on chromosome 11, which replaces glutamic acid in position 6 of beta-globin chain by valine, producing an abnormal haemoglobin S (HbS) molecule [1,2]. The term Sickle Cell Disease applies to those patients who have at least one abnormal $\mathrm{HbS}$ chain and another abnormal beta chain. If the second abnormal beta chain is also an HbS chain then the patient is considered to be homozygous HbSS. Alternatively, other abnormal haemoglobins like HbC or beta-thalassemia result from HbSC and HbS-beta $(\mathrm{S} \beta)$, respectively.

Deoxygenation of $\mathrm{HbS}$ results in the aggregation of abnormal haemoglobin molecules into long chains that tend to distort the red blood cell membrane into a rigid sickle shape [1,2] (Figure 1). The consequences are obstruction of the microcirculation, ischaemia, and infarction [1,2]. Anaemia results from the rapid removal of abnormal red blood cells by the reticuloendothelial system, which reduces the red cell life span to one tenth of its normal duration. Thus, disease manifestations can be roughly attributed to two phenomena: haemolysis and vaso-occlusion. Haemolytic anaemia occurs in all forms of SCD and results from the destruction of the sickled cells, cell dehydration and direct membrane damage by rigid haemoglobin polymers [2]. Vasoocclusion is due to entrapment of the sickled cells in the microcirculation that leads to tissue ischaemia and damage in almost all 
organs [2]. Several organs can be affected with alterations in the central nervous system, bone and joints, cardiovascular system, respiratory system, gastrointestinal tract and kidneys which increase morbidity and mortality in this group of patients [1].

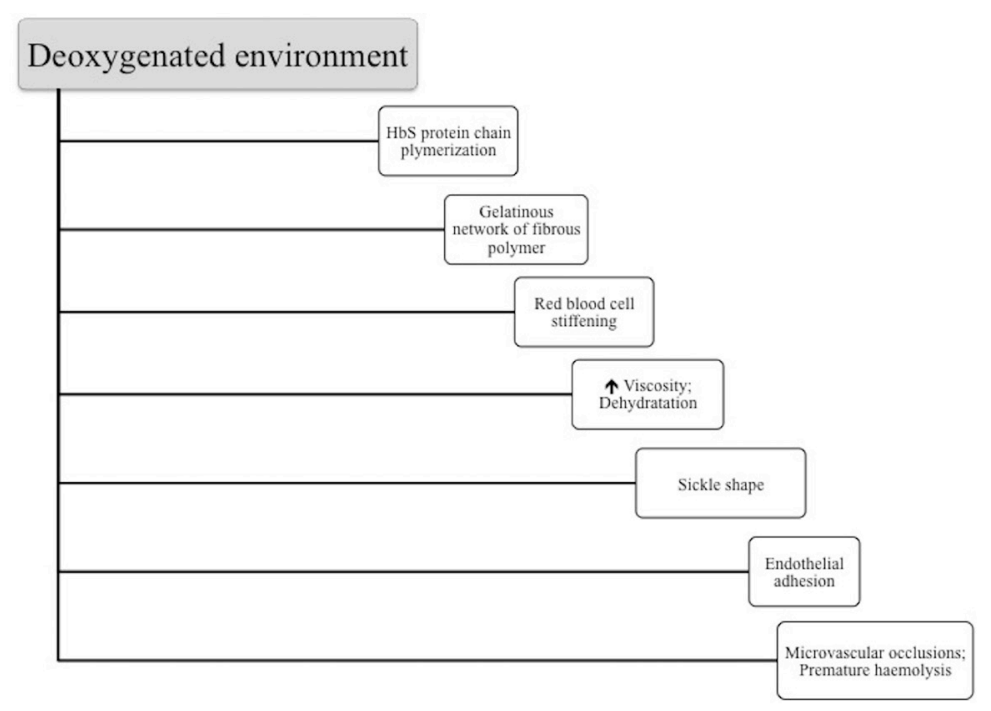

Figure 1: SCD pathophysiology [1,2] - The abnormal HbS protein chain polymerizes reversibly in deoxygenated environment into a gelatinous network of fibrous polymers that stiffen the RBC (red blood cell) membrane, increases the viscosity, and causes dehydration resulting in a sickle shape. These cells are abnormally sticky causing microvascular occlusions and premature haemolysis

Osteo-articular involvement in SCD is mostly represented by marrow replacement and hyperplasia, osteonecrosis, osteomyelitis and arthritis.

Bones are the second most affected organ by SCD, after the spleen [3]. Despite the importance of osteo-articular involvement in SCD, these complications are still understudied and the pathophysiology of these changes is not fully understood.

The most frequent complications that require hospitalization in SCD patients are painful vaso-occlusive crises (VOC) and osteomyelitis [4].

This review addresses acute and long-term osteo-articular complications in SCD, with particular emphasis on differentiating infection from infarction (osteonecrosis), where imaging is playing a crescent role on the acute bone pain diagnosis algorithm.

\section{Materials and Methods}

We performed a retrospective study to illustrate the osteo-articular manifestations associated with SCD in our institution, in children with at least one Haematology appointment in 2013 and 2014. Osteo-articular manifestations with imaging findings were reported in 28 out of 97 patients ( 23 homozygous - SS patients and 5 composite heterozygous - SC, S $\beta$ ) with equal gender distribution. These cases were reviewed and 11 major manifestations were depicted (infection and avascular necrosis). The remaining patients presented 17 minor manifestations such as effects of intramedullary marrow with replacement and hyperplasia as well as growth effects. All the imaging examinations were first analysed by senior doctors specialized on Paediatrics Radiology without following any specific protocol. During the study all the imaging findings were reviewed by a junior doctor and a senior doctor specialized in musculoskeletal radiology.

\section{Results}

\section{Marrow replacement and hyperplasia}

Increased destruction of red blood cells and consequent anaemia causes persistence and expansion of the red (haematopoietic) marrow [2]. Marrow hyperplasia results in widening of the medulla and subsequent cortical thinning, resulting in coarsening of the normal trabecular pattern with loss of corticomedullary differentiation (Figure 2). This process may cause the bone to appear osteopenic and prone to pathologic fractures [1].

Cranial diploe prominence is observed in the skull, as well as flattening of the external and internal laminae and increased thickness of the frontal and parietal bones (Figure 2). The best example of bone softening is seen in the vertebral bodies, where the end plates assume a smooth concavity described as fish mouth vertebra [5] (Figure 2). On T1 weighted image (WI), normal fatty marrow shows high signal intensity, while haematopoietic red marrow is low in signal (Figure 2).

Extramedullary haematopoiesis is also described in SCD however, we did not find it in our series. The most common site is the liver, but the spleen may also be affected, and soft-tissue haematopoietic masses may develop in the thorax, adrenal glands and the skin. 


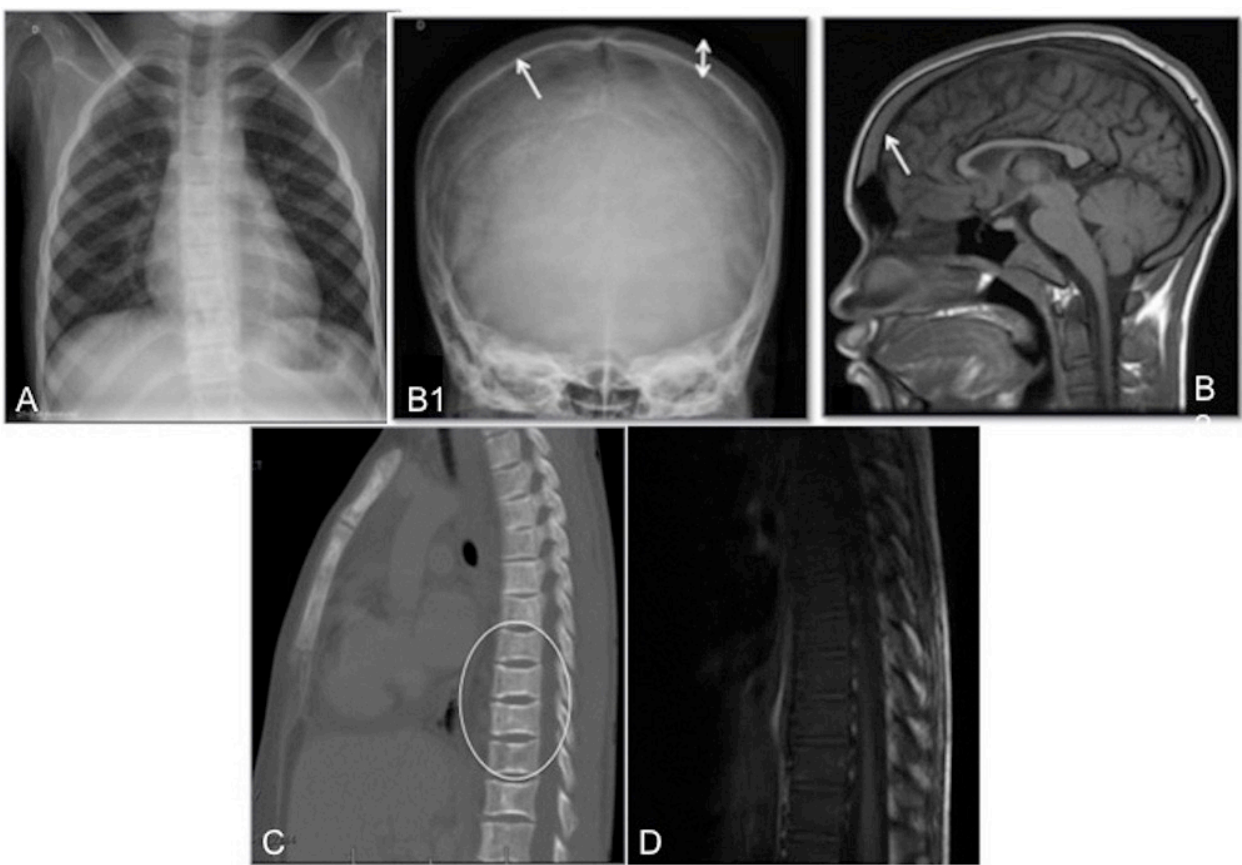

Figure 2: (A) Effects of medullary hyperplasia. Postero-anterior chest radiograph of a 14 year-old HbSS girl, showing coarsening of trabecular pattern with loss of corticomedullary differentiation particularly on the ribs; (B) Bone marrow hyperplasia within the skull vault; (B1) Postero-anterior radiograph from a 10 year-old Hb SS girl. There is widening of the diploic space (arrows); (B2) T1W Sagittal MR image of the same patient, the medullary cavity is widened with low signal intensity; (C) Thoracic CT on the sagittal bone window view of an 18 year-old HbSS girl. Smooth concavity of the vertebral endplates at multiple levels from bone softening (fish mouth vertebrae). Note patchy sclerosis of the vertebrae and sternum, caused by medullary infarction; (D) T1W MRI sagittal view of the spine in a 12 year-old HbSS boy, shows low signal intensity indicative of cellular (red) marrow hyperplasia

\section{Bone Infarcts}

The abnormal red cell shape causes blood stasis and cell sequestration in the bone capillaries resulting in ischaemia and tissue hypoxia, which in turn, worsen the sickling process. Bone infarction can take place in the diaphysis causing medullary infarcts, and in both epiphyses causing avascular necrosis. This can present as the classical painful bone crisis, although it may also be clinically silent and an incidental finding on radiography [6].

Initial radiographs are usually normal in acute infarction [7]. Chronic infarcts demonstrate intramedullary lucency and patchy sclerosis with or without periosteal reaction, depending if cortical bone is also affected. MRI is more sensitive than CT and plain radiographs in the detection of bone infarcts. On T2 WI, infarction is seen as an area of high signal intensity. Infarcts may also show post gadolinium peripheral enhancement and soft tissue changes, complicating the differential diagnosis with osteomyelitis in the acute setting [8] (Figure 3 and 4).

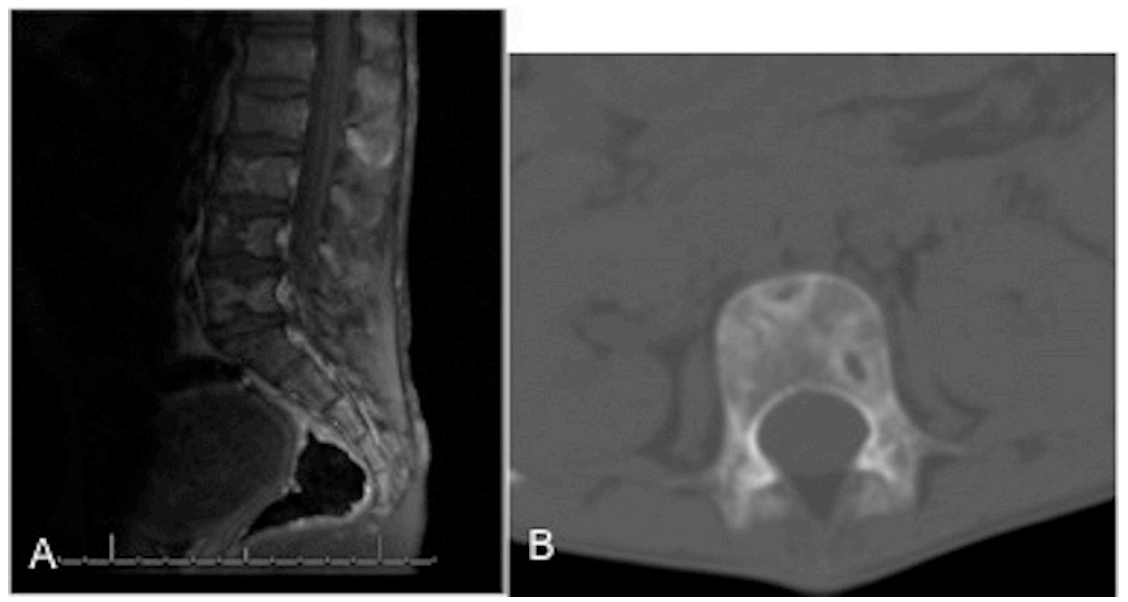

Figure 3: (A) T1W FS MRI, sagittal view of a 12 year-old $H b S \beta^{\circ}$ boy with vertebral infarction at different levels, on L3 L4 and L5 showing abnormal heterogeneous signal intensity, with collapse of the vertebral bodies; (B) CT Axial view on bone window of same patient showing patchy sclerosis of the vertebral body consistent with chronic infarction

In young children, infarction occurs within the small bones of the hand and feet resulting in painful dactylitis termed "handfoot" syndrome. Sickle cell dactylitis is common between the ages of 6 months and 2 years but is rare after the age of 6 due to regression of red marrow in these areas with increasing age [9] (Figure 4). In our series we only depicted 2 cases of dactylitis, both in homozygous HbSS patients, and this low number is due to imaging not being routinely performed in dactylitis. 

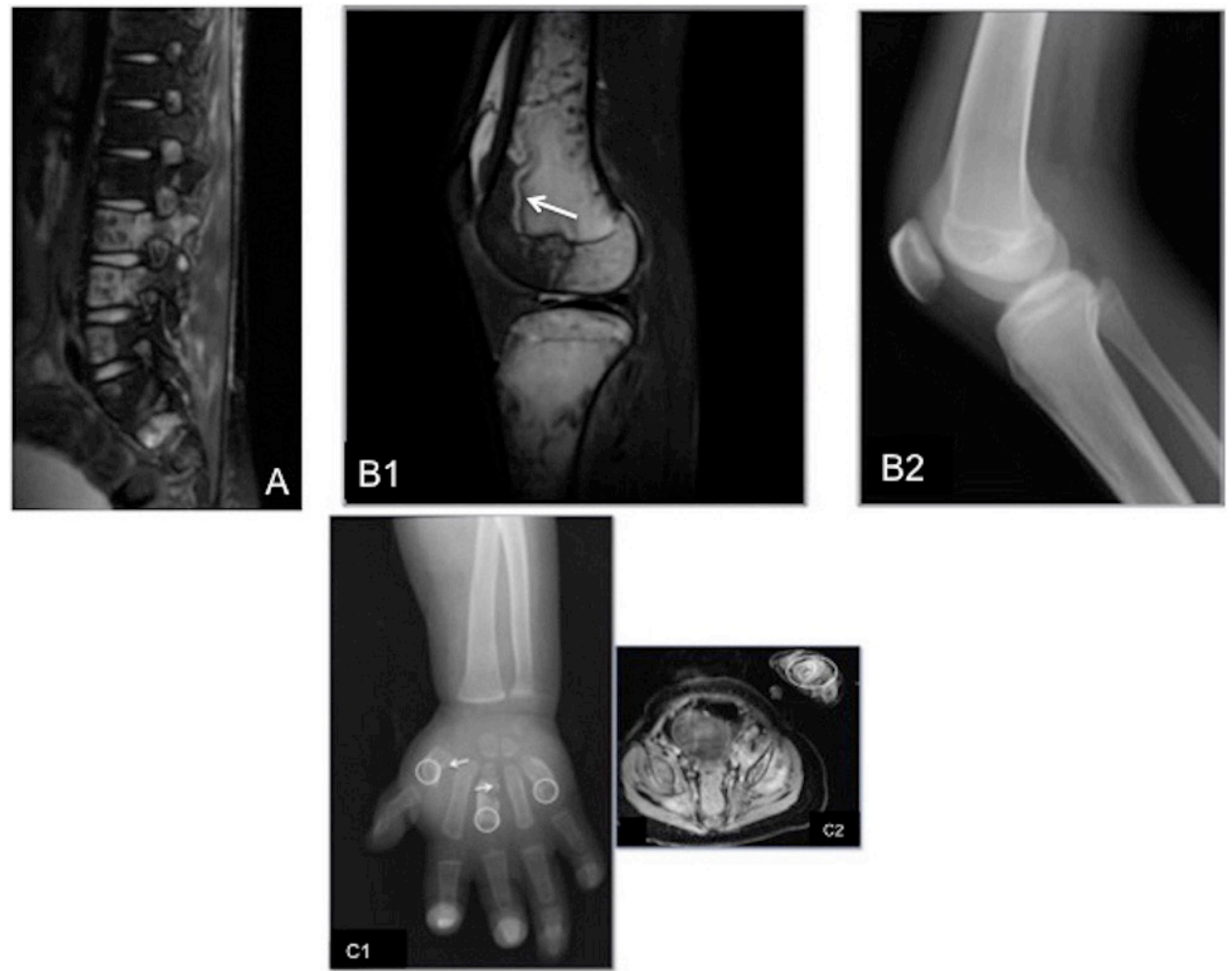

Figure 4: (A) T2W FS MRI, lumbar sagittal view of a10 year-old homozygous HbSS boy, revealing high signal intensity at L2, L3 and L4 vertebral bodies consistent with osseous oedema in the setting of acute bone pain and acute infarction; (B1) DP FS MRI of the right knee on sagittal view of a 13 year-old HbSS boy with acute right knee pain, where we can see a serpiginous double line (white arrow) at the distal femur and proximal tibia not affecting the articular surface, consistent with medullary infarcts; (B2) Knee plain radiograph on lateral projection of the same patient, performed at the same time of the MRI, no radiographic changes were depicted; (C1) Left hand plain radiograph on AP projection of a 5 month HbSS boy, with periosteal new bone formation along the diaphysis of the first and third metacarpal (arrow) and lucencies (circles) in keeping with early destructive lesions consistent with dactylitis; (C2) T1FS MRI, with hyperintensity at the base of the third metacarpal (circle) consistent with sequestered blood in the marrow space in the subacute phase of an infarction

Another pattern of bone infarction, as previously referred is epiphyseal involvement. This is commonly known as avascular necrosis $(\mathrm{AVN})$. In spite of $50 \%$ of patients with SCD developing AVN by the age of 35 years, it was hypothesised that, the lower prevalence in young children could be due to the contribution of synovial fluid in epiphyseal nutrition [10]. Again, initial radiographs are normal but as osteonecrosis progresses, changes become more evident. Early radiographic signs include lucency and sclerosis within the epiphysis. Subsequently, crescent-shaped subchondral lucencies develop and later, depression of the articular surface with collapse and eventually fragmentation may occur (Figure 5 and 6). The earliest signs of AVN are seen on MRI, particularly T2 WI with fat supressed sequences showing regions of high signal intensity indicative of bone marrow oedema. A serpiginous line is classically seen, consisting in a hyperintense inner border and hypointense periphery. This double line results from the high signal intensity inflammatory response of bone with granulation tissue, inside the low signal intensity reactive bone interface [11] (Figure 7). The prevalence and incidence of osteonecrosis in SCD was studied by Milner in 2590 patients. The femoral head was most commonly involved followed by the humeral head. Furthermore, patients with HbSS and alpha thalassemia were at higher risk of developing this complication [12].

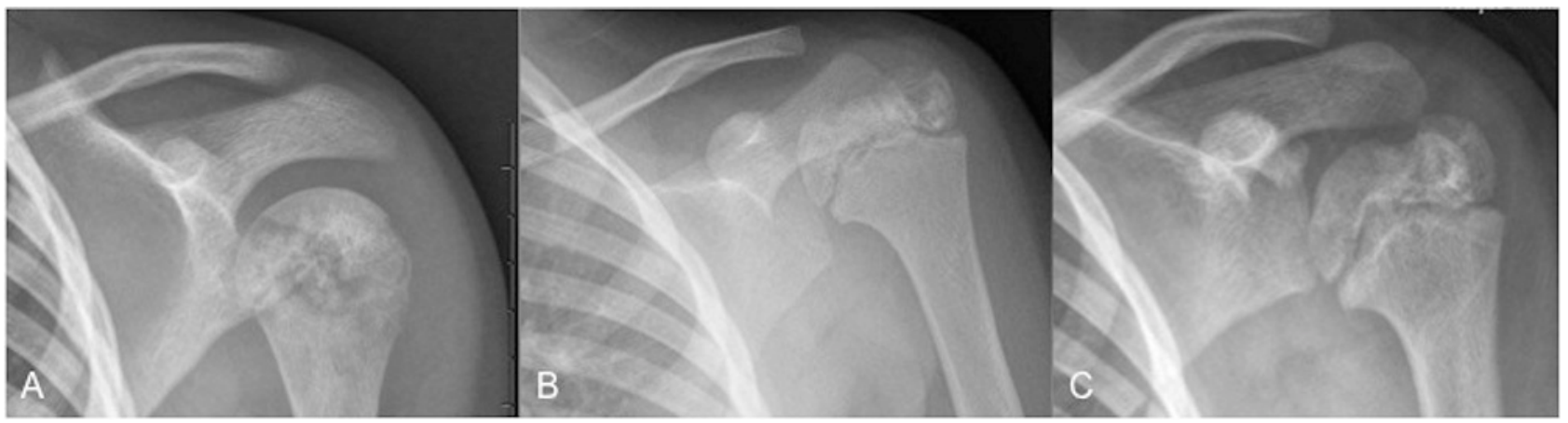

Figure 5: Plain radiographs on antero-posterior projection of a 9 year-old HbSS/ alpha thalassemia boy showing left humeral head AVN progress within 4 months. In (A) Signs of epiphysial lucencies; (B) Subcondral lucencies with depression of articular surface; (C) Collapse of the articular surface with flattening of the humeral head

Seven cases of avascular necrosis were found in our series. The gender ratio was 5 females : 2 males. Six cases were on the femoral head and one case on the humeral head. Four of the ones with femoral head necrosis required surgery. 


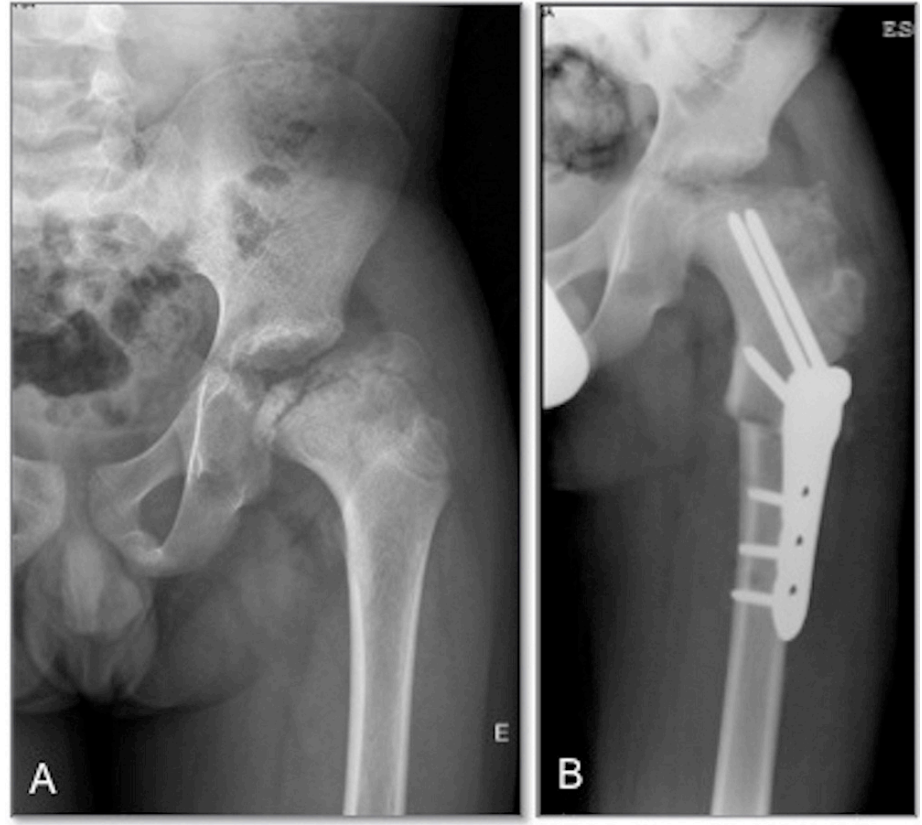

Figure 6: (A) Plain radiographs in AP projection of a10-year old HbSS/alpha thalassemia boy presenting with left femoral head osteonecrosis with advanced degenerative changes due to the collapse of the femoral head; (B) valgisation osteotomy was performed
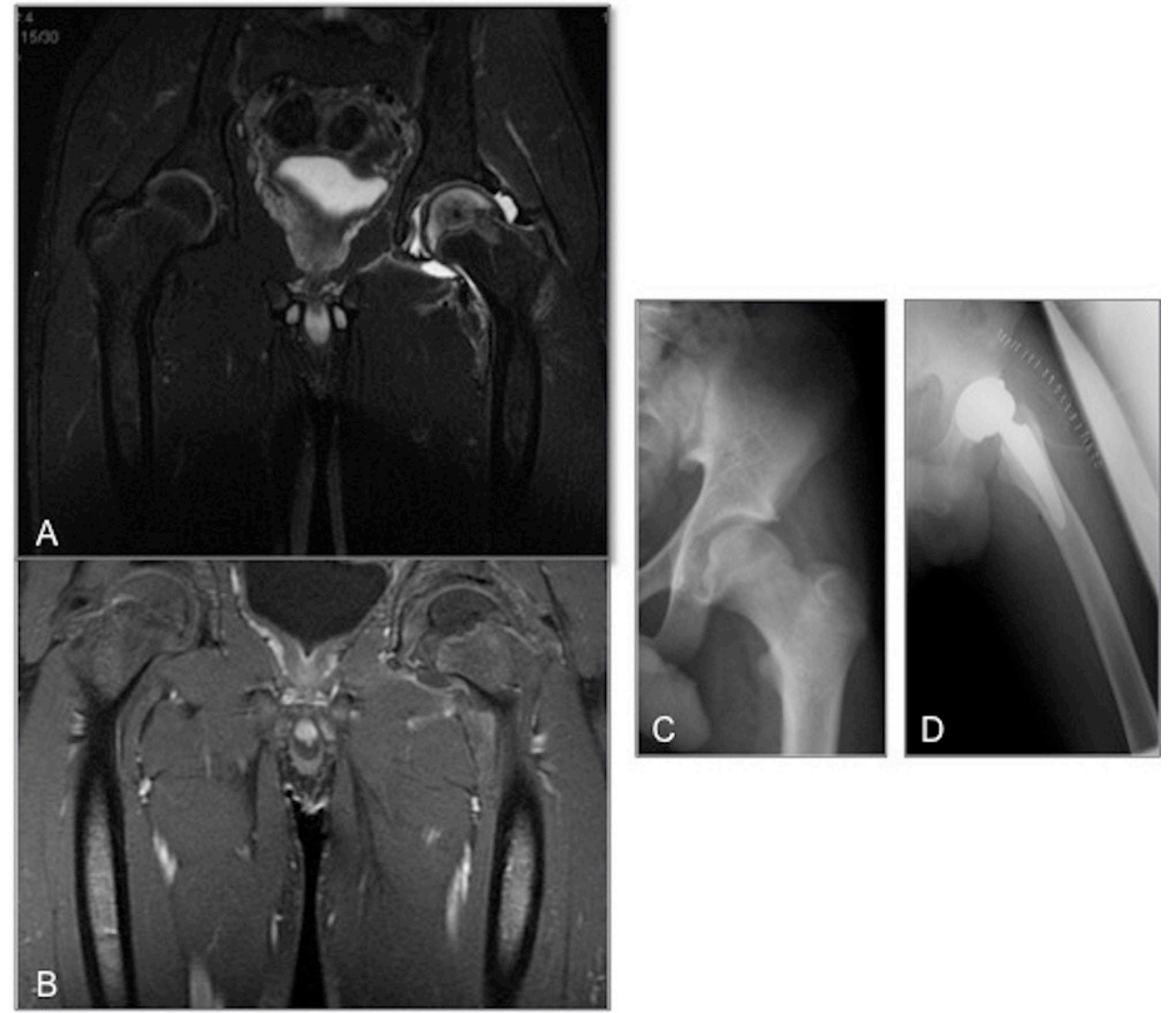

Figure 7: Twelve year-old HbSS boy, presenting with acute pain on the left hip (A) T2W FS MRI, coronal view, high signal intensity on the left femoral head consistent with osseous oedema; (B) T1 W FS post gadolinium, coronal view, no enhancement on post contrast is depicted on the left femoral head in keeping with infarction; (C) Plain radiograph on AP projection, several months later, flattening of the femoral head; (D) Total hip arthroplasty was performed

\section{Osteomyelitis}

Osteomyelitis is a serious complication of SCD and usually occurs in long bones e.g. femur, tibia, and humerus [1]. The high frequency of infection in patients with SCD is due to a number of factors. Hyposplenism results in a degree of immunosuppresion [5] and infarcted or necrotic bone is a good environment for bacterial growth [3]. In addition, multiple hospital admissions may increase the patient's exposure to certain bacterial pathogens [5] (Figure 8). The most common infectious agents of osteomyelitis in patients with SCD are Salmonella spp and Staphylococcus aureus [1]. 


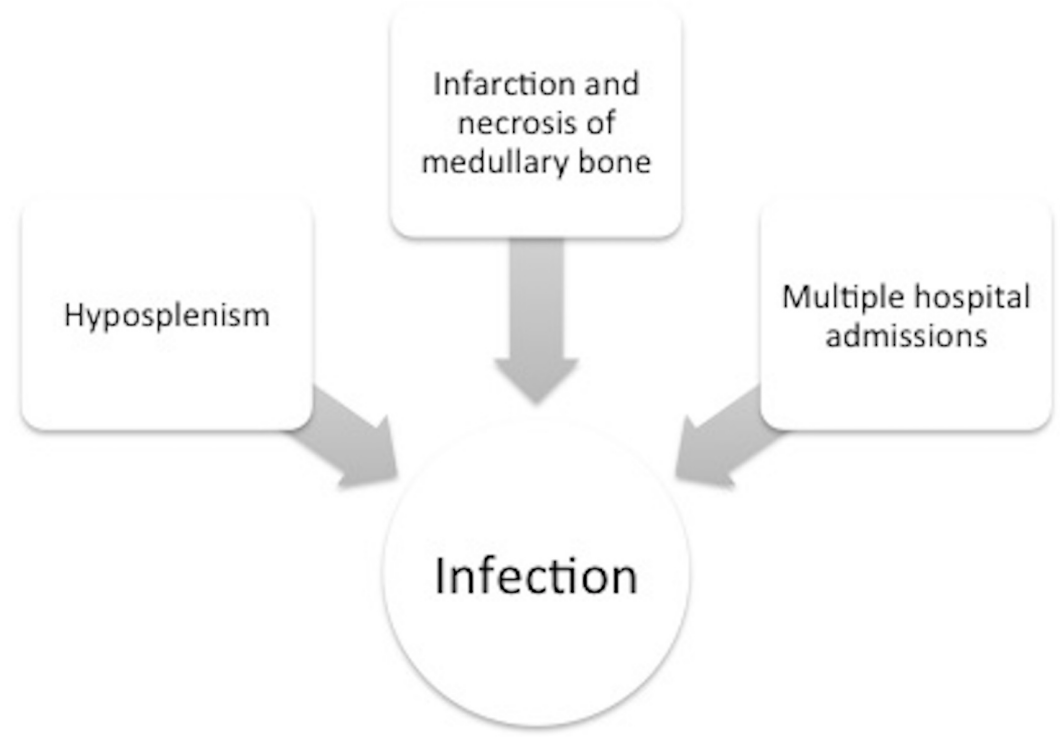

Figure 8: Factors that may contribute to high frequency of infection in SCD $[1,3,5]$

Osteomyelitis occurs in $18 \%$ while septic arthritis occurs in $7 \%$ of patients with SCD according to a previous study [13].

Six cases of infection were observed in our series, 5 cases of osteomyelitis (one of them a multifocal osteomyelitis) and 1 case of arthritis.

The classical clinical findings of pain, fever and raised inflammatory markers can also be seen in infarction, which can cause diagnostic difficulty in the acute setting [14]. All available biological material should be sent for microbiological analysis as soon as possible, including blood, joint effusion aspirate and subperiosteal fluid [14]. Sonography is primarily performed to localize the fluid collection and guide the aspiration to obtain a sample for bacteriological analysis $[14,15]$. The specificity of needle aspiration is limited for only 60 to $75 \%$ of tissue biopsies and $70 \%$ of bone or joint aspirates are positive for infection [15]. It has also been demonstrated that only 50 to $58 \%$ of patients with acute osteomyelitis have positive blood cultures, probably because of prior broad-spectrum antibiotic therapy. Conversely not all patients with positive blood cultures and acute bone pain have acute osteomyelitis [15].

Plain film findings of osteomyelitis include osteopenia, periosteal reaction with or without associated cortical destruction, sinus tract formation and soft tissue extension. There are features such as osteopenia and periosteal reaction that are not specific of osteomyelitis and can also be seen in acute bone infarction. But the main question with plain films is that the earliest changes may not be evident for 8-10 days from the beginning of the clinical complains.

US have been reported to have high sensitivity in diagnosing osteomyelitis in children with SCD. In a recent study it was reported that sub-periosteal fluid collection with a diameter above $4 \mathrm{~mm}$ was a strong indicator of osteomyelitis [16].

MR imaging is an increasingly useful tool in the diagnosis of osteomyelitis in SCD. On T1 sequences osteomyelitis has low signal intensity. Interpretation of these findings should be careful because areas of red marrow will also have low signal intensity. T2 WI fat saturated sequences may show fluid collections as an area of high signal intensity within the bone marrow. These sequences are also useful for demonstrating the communication of soft-tissue fluid collections with medullary fluid collections through cortical defects. Osteomyelitis will also show areas of post-gadolinium enhancement. This will tend to be more diffuse than in infarction. It has been proposed that the degree of thickness of rim enhancement differs between osteomyelitis and acute infarction [8]. Thus, in osteomyelitis, there is a thick irregular rim of enhancement whereas acute infarction results in thin linear rim enhancement [8] (Figure 9, 10, 11 and 12).

There is no reference standard for diagnosing sickle cell osteomyelitis, and even the culture of biopsy specimens, as previously mentioned, is not completely reliable [15]. The lack of a reference standard makes the comparison of imaging modalities difficult. Therefore, the interpretation of radiologic findings must be part of a careful ongoing multidisciplinary assessment. However it must be emphasized that acute bone infarction is about 50 times more frequent than acute osteomyelitis [4].

Recently it was proposed that acute bone infarcts in SCD are caused by sequestration of red blood cells in the bone marrow [17]. Furthermore it was also suggested that $\mathrm{T} 1$ fat saturated sequence alone is diagnostic for acute bone infarcts, when high signal intensity is depicted in the acute pain setting. Contrast enhancement sequences can be performed if no high signal intensity is depicted on T1 fat saturated in order to diagnose or rule out osteomyelitis (Figure 13).

Septic arthritis is less common than osteomyelitis. It often occurs in the setting of vaso-occlusion and bone infarction. Both US and MRI are useful and may include features like joint effusion and peri-synovial oedema. 

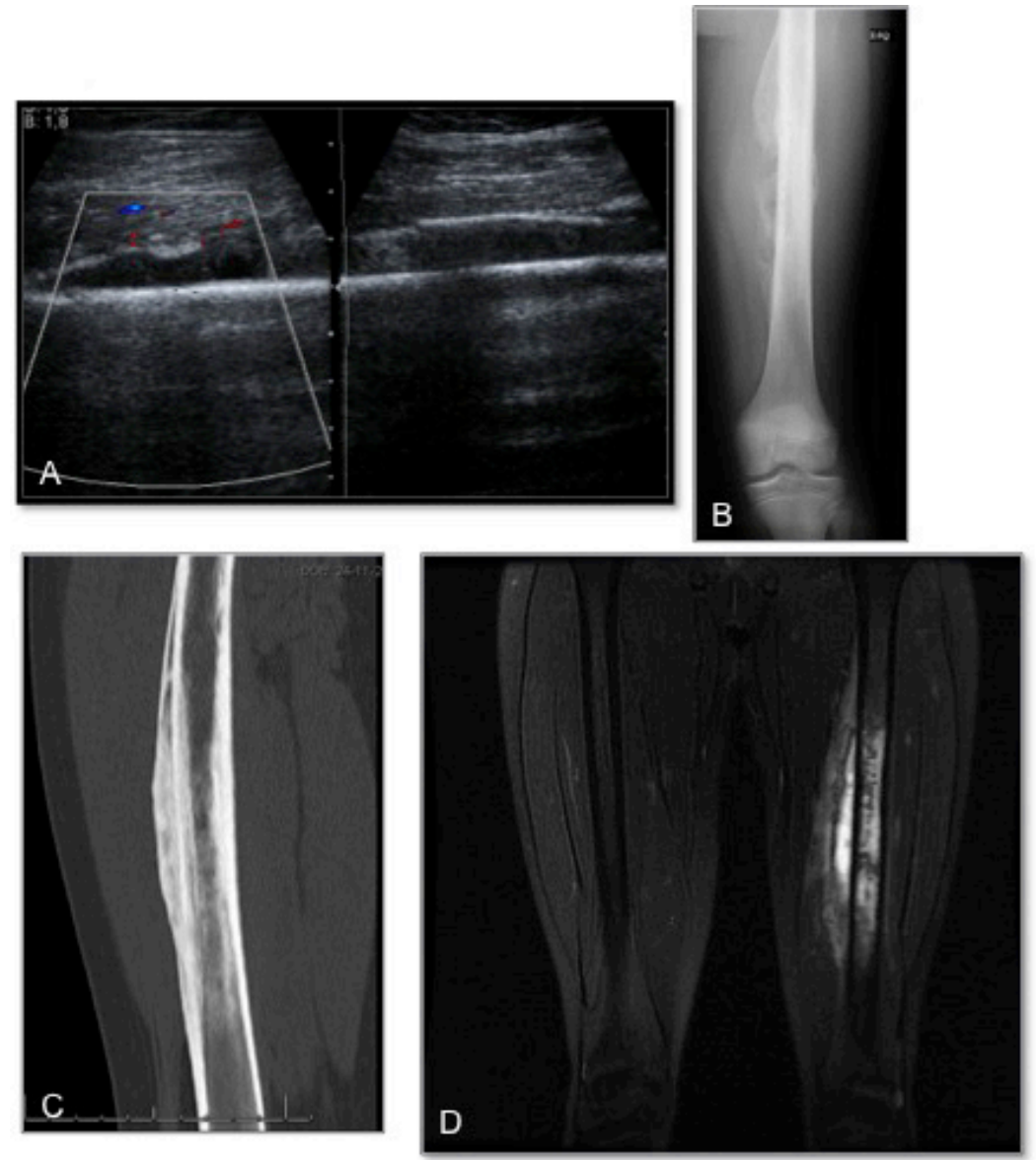

Figure 9: Osteomyelitis of the left femur in a 11 year-old HbSS girl, no agent was identified (A) Sonography of the left thight, where there is a subperiosteal liquid collection in the femur with a depth over $4 \mathrm{~mm}$. There is also some cortical irregularity and subcutaneous tissue thickening; (B) Plain radiograph on AP projection showing subperiosteal reaction; (C) CT - Sagittal reconstruction on bone window depicting a subperosteal collection and thickening of the cortical bone; (D) Coronal T2W FS MRI, obtained at a follow-up, showing medullary high signal intensity as well as soft and periosteal collections, later found to be an abscess

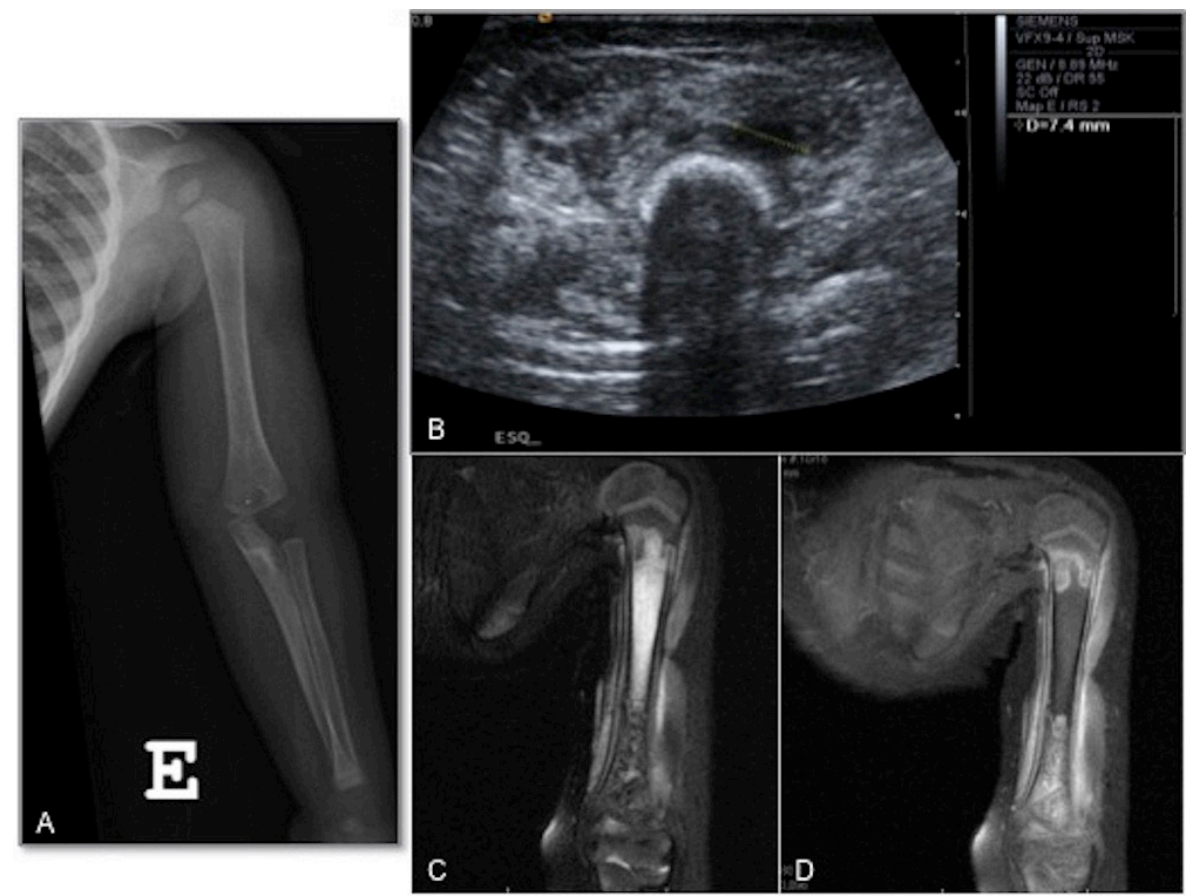

Figure 10: Osteomyelitis of the left humerus in a 1 year-old HbSC boy. No agent was isolated. (A) Plain radiograph of the left arm with no pathological features; (B) Sonography on a transversal view, with periosteal collection on the left humerus with $7 \mathrm{~mm}$ diameter; (C) T2-weighted fat supressed MR image, showing high signal intensity on the humerus diaphysis in keeping with osseous oedema; note also the high signal intensity of the surrounding soft tissues; (D) T1-weighted fat supressed MR image post gadolinium showing geographic enhancement at the proximal aspect of humerus diaphysis and also in the distal portion involving the distal epiphysis, consistent with an osteomyelitis enhancement pattern 

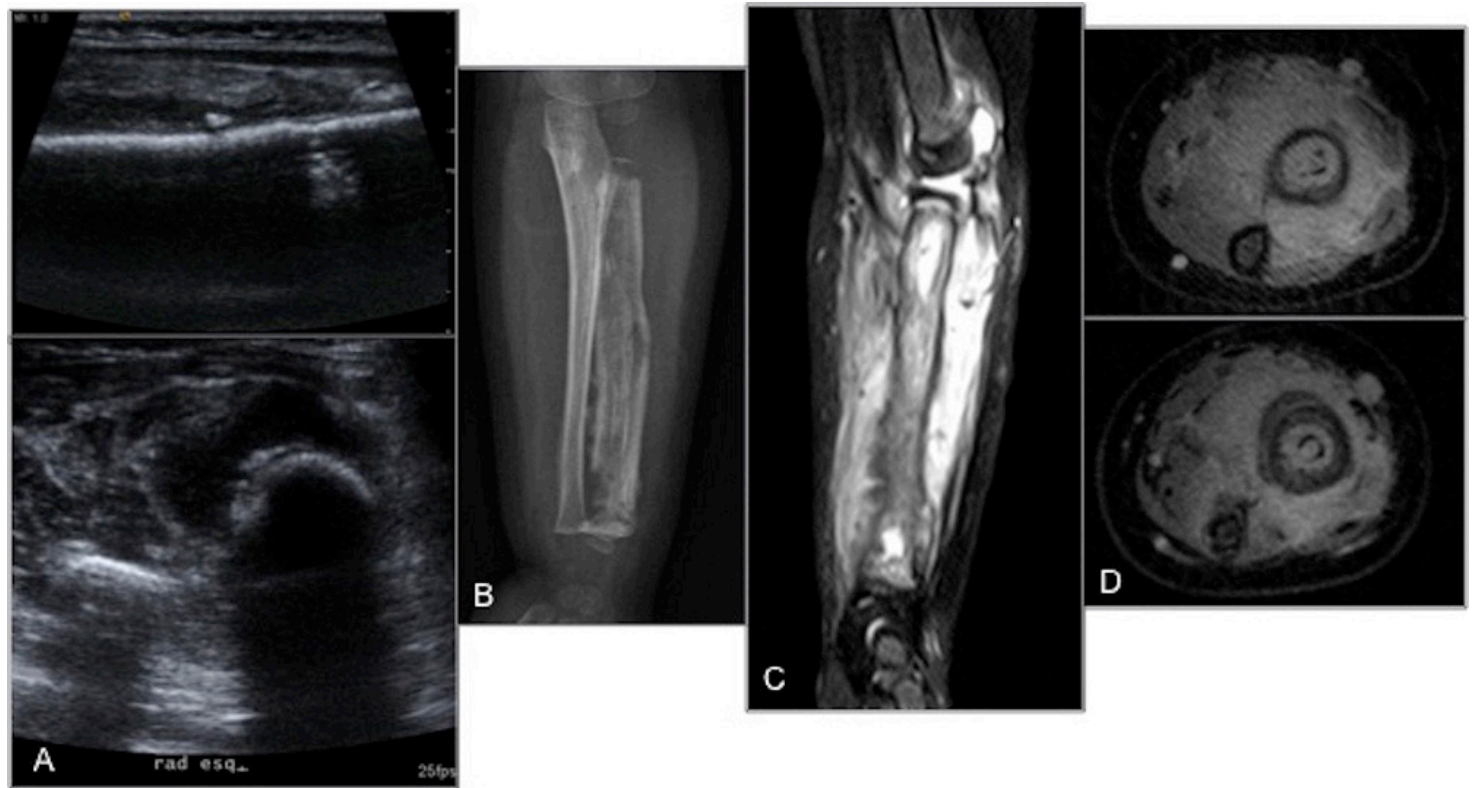

Figure 11: Osteomyelitis of the left radius in a 2 year-old HbSS/alpha thalassemia girl, no agent was isolated. (A) Sonography revealing a liquid subperiosteal collection, with cortical irregularity; (B) Plain radiography on AP projection, showing multifocal bone destruction in the radius characterized by medullary hyperlucency with cortical irregularities; (C) T2 weighted fat supressed MR image, shows high signal intensity within the radius medullary cavity and liquid collections of the surroundings soft tissues; (D) T1 weighted fat supressed MR image post gadolinium showing a thick and irregular enhancement on the medullary radial cavity

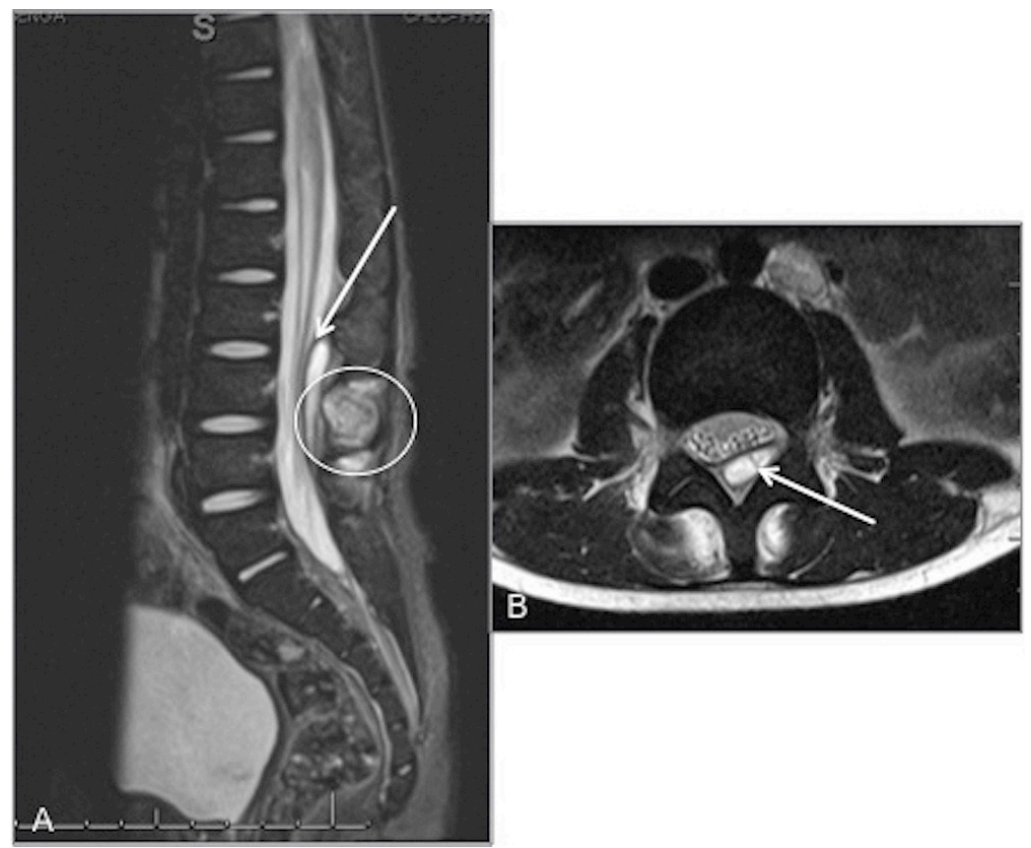

Figure 12: Osteomyelitis of the posterior L3 arch in a 5 year-old HbSS boy. T2 weighted MR image (A) Sagittal View and (B) Axial view, showing high signal intensity on the posterior L3 arch (circle). There is also an epidural collection (arrow)

\section{Soft tissue abnormalities}

Occlusion of vessels leads to inflammation, oedema and myonecrosis [14]. Fluid collections, hematomas and fat necrosis may occur in soft tissue. No cases of isolated soft tissue abnormalities alone were encountered in our imaging series.

US and MRI have a good sensitivity for identifying soft-tissue changes. On MRI, high signal intensity within muscle on T2 WI is the main feature of soft tissue involvement [14].

\section{Growth effects}

Patients with SCD may have reduced height [1]. This is believed to be due to bone marrow hyperplasia. Bones are generally shorter due to epiphyseal shortening secondary to ischaemia/infarction and vascular compromise to the growth plate [14]. Premature closure of growth plates also occurs [14]. 


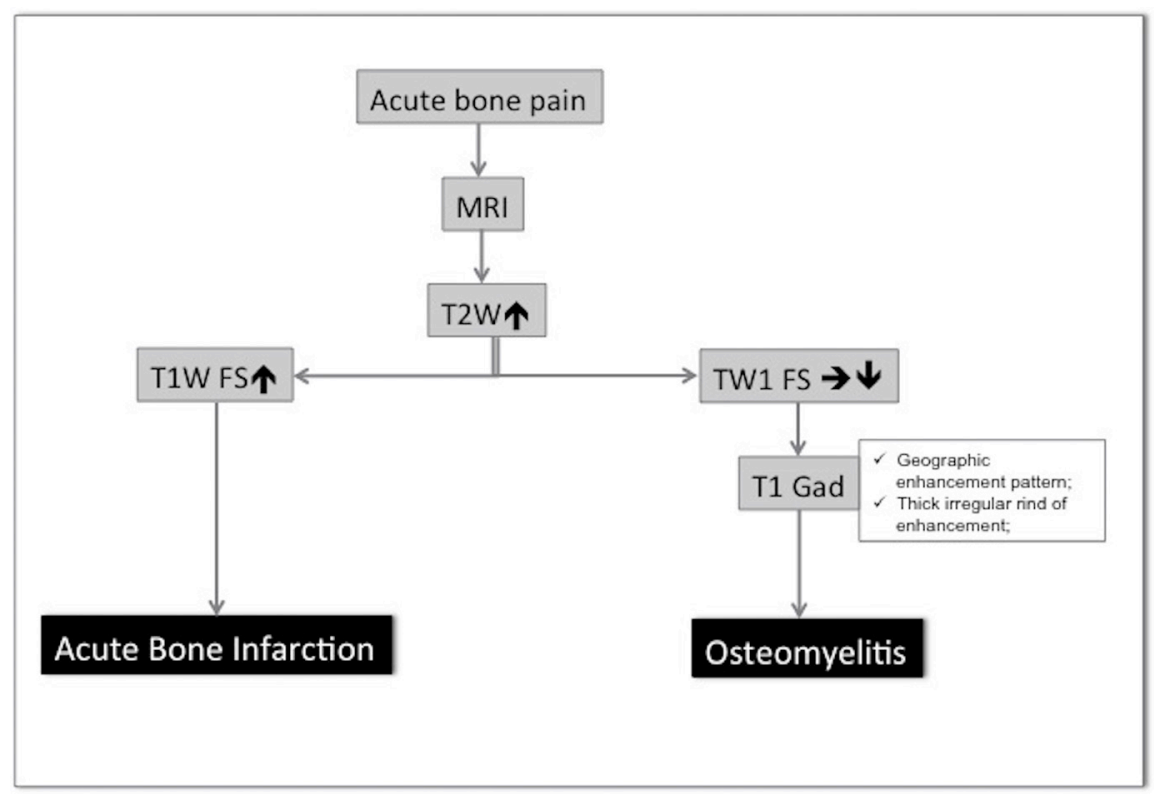

Figure 13: Management algorithm of acute bone pain in SCD [8,17] - In strong equivocal cases of acute bone pain an MRI should be performed. In the presence of high signal intensity on T2 and T1 FS sequences acute bone infarction should be diagnosed. In the setting of high signal intensity on T2 and a low or isointense signal on T1 FS, contrast enhancement should be performed, to confirm or exclude the diagnosis of osteomyelitis

\section{Conclusion}

Awareness of the imaging features of SCD is important for early diagnosis and management of musculoskeletal complications.

Acute osteomyelitis may be particularly difficult to distinguish from bone infarction, and MRI could be an important tool in these cases.

\section{References}

1. Wang WC (2009) Sickle cell anemia and other sickling syndromes In: Wintrobe’s Clinical Hematology (12 ${ }^{\text {th }}$ Edn) Philadelphia: Lippincott Williams and Wilkins, USA.

2. Schnog JB, Duits AJ, Muskiet FA, ten Cate H, Rojer RA, et al. (2004) Sickle cell disease; a general overview. Neth J Med 62: 364-74.

3. da Silva GB Jr, Daher EDF, da Rocha FAC (2012) Osteoarticular involvement in sickle cell disease. Rev Bras Hematol Hemoter 34: 156-64.

4. Almeida A, Roberts I (2005) Bone involvement in sickle cell disease. Br J Haematol 129: 482-90.

5. Ganguly A, Boswell W, Aniq H (2011) Musculoskeletal Manifestations of Sickle Cell Anaemia: A Pictorial Review. Anemia 8: 1-9.

6. Ware HE, Brooks AP, Toye R, Berney SI (1991) Sickle cell disease and silent avascular necrosis of the hip. J Bone Joint Surg Br 73: 947-9.

7. Madani G, Papadopoulou AM, Holloway B, Robins A, Davis J, et al. (2007) The radiological manifestations of sickle cell disease. Clinical Radiology 62: 528-38.

8. Umans H, Haramati N, Flusser G (2000) The diagnostic role of gadolinium enhanced MRI in distinguishing between acute medullary bone infarct and osteomyelitis. Magn Reson Imaging 18: 255-62.

9. Stevens MC, Padwick M, Serjeant GR (1981) Observations on the natural history of dactylitis in homozygous sickle cell disease. Clin Pediatr 20: 311-7.

10. Adekile AD, Grupta R, Yacoub F, Sinan T, Al Bloushi M, et al. (2001) Avascular necrosis of the hip in children with sickle cell disease and high Hb F:magnetic resonance imaging findings and influence of alpha-thalassemia trait. Acta Haematol 105: 27-31.

11. Stroller DW, Tirman PFJ, Bredeller MA, Beltran S, Branstetter RM III, et al. (2004) The hip. In: Diagnostic imaging orthopaedics. Salt Lake City, Utah, USA.

12. Milner PF, Kraus AP, Sebes JI, Sleeper LA, Dukes KA, et al. (1991) Sickle cell disease as cause of osteonecrosis of the femoral head. N Engl J Med 325: 1476-81.

13. Bahebeck J, Atangana R, Techa A, Monny-Lobe M, Sosso M, Hoffmeyer (2004) Relative rates and features of musculoskeletal complications in adult sicklers. Acta Orthopaedica Belgica 70: 107-11.

14. Lonergan GJ, Cline DB, Abbondanzo SL (2001) Sickle cell anemia. Radiographics 21: 971-94.

15. Skaggs DL, Kim SK, Greene NW, Harris D, Miller JH (2001) Differentiation between bone infarction and acute osteomyelitis in children with sickle cell disease with use of sequential radionuclide bone-marrow and bone scans. J Bone Joint Surg Am 83-A: 1810-3.

16. Inusa BP, Oyewo A, Brokke F, Santhikumaran G, Jogeesvaran KH (2013) Dillemma in Differentiating between acute osteomyelitis and bone Infarction in children with Sickle Cell Disease: The Role of Ultrasound. Plos One 8: 10.1371/journal.pone.0065001.

17. Jain R, Sawhney S, Rizvi SG (2008) Acute bone crisis in sickle cell disease: the T1 fat-saturated sequence in differentiation of acute bone infarcts from acute osteomyelitis. Clin Radiol 63: 59-70. 


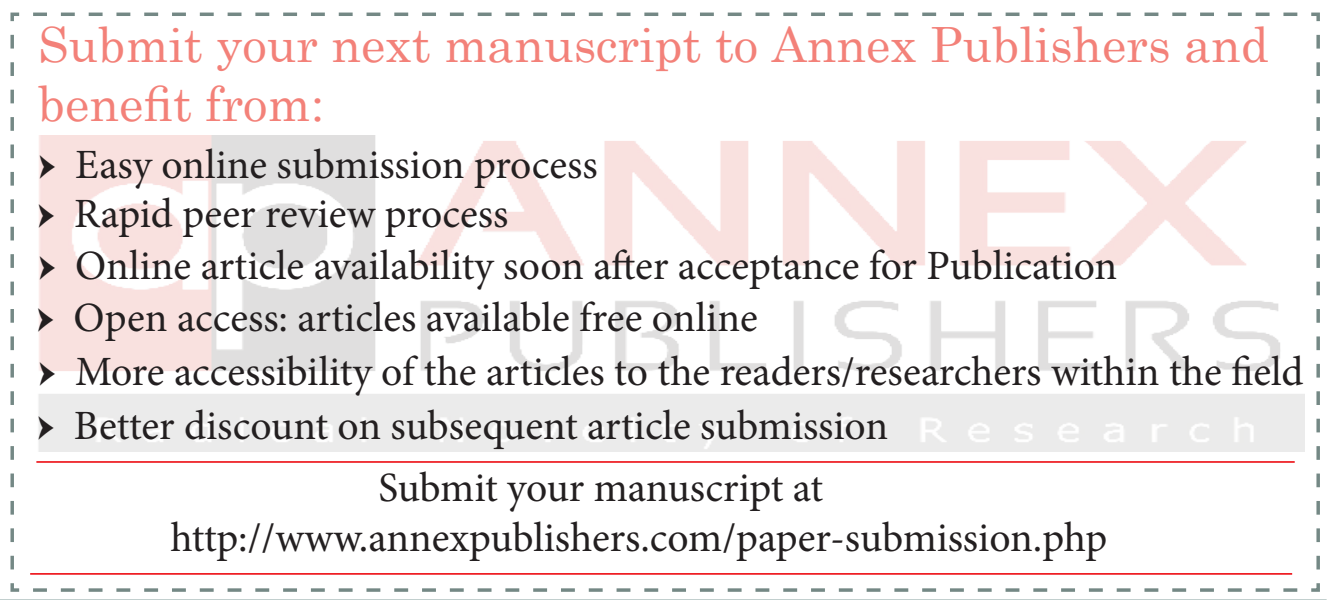

